\title{
Vertical-Cavity In-plane Heterostructures: Physics and Applications
}

\author{
Taghizadeh, Alireza; Mørk, Jesper; Chung, II-Sug
}

Published in:

Applied Physics Letters

Link to article, DOI:

$10.1063 / 1.4935084$

Publication date:

2015

Document Version

Publisher's PDF, also known as Version of record

Link back to DTU Orbit

Citation (APA):

Taghizadeh, A., Mørk, J., \& Chung, I-S. (2015). Vertical-Cavity In-plane Heterostructures: Physics and Applications. Applied Physics Letters, 107(18), [181107]. https://doi.org/10.1063/1.4935084

\section{General rights}

Copyright and moral rights for the publications made accessible in the public portal are retained by the authors and/or other copyright owners and it is a condition of accessing publications that users recognise and abide by the legal requirements associated with these rights.

- Users may download and print one copy of any publication from the public portal for the purpose of private study or research.

- You may not further distribute the material or use it for any profit-making activity or commercial gain

- You may freely distribute the URL identifying the publication in the public portal

If you believe that this document breaches copyright please contact us providing details, and we will remove access to the work immediately and investigate your claim 


\title{
Vertical-cavity in-plane heterostructures: Physics and applications
}

\author{
Alireza Taghizadeh, Jesper Mørk, and II-Sug Chung ${ }^{\text {a) }}$ \\ Department of Photonics Engineering (DTU Fotonik), Technical University of Denmark, DK-2800 Kgs. \\ Lyngby, Denmark
}

(Received 25 August 2015; accepted 22 October 2015; published online 2 November 2015)

\begin{abstract}
We show that in-plane (lateral) heterostructures realized in vertical cavities with high contrast grating reflectors can be used to significantly modify the anisotropic dispersion curvature, also interpreted as the photon effective mass. This design freedom enables exotic configurations of heterostructures and many interesting applications. The effects of the anisotropic photon effective mass on the mode confinement, mode spacing, and transverse modes are investigated. As a possible application, the method of boosting the speed of diode lasers by engineering the photon-photon resonance is discussed. Based on this platform, we propose a system of two laterally coupled cavities, which shows the breaking of parity-time symmetry in vertical cavity structures. (C) 2015 AIP Publishing LLC. [http://dx.doi.org/10.1063/1.4935084]
\end{abstract}

The vertical cavity is a rich platform for fundamental physics studies of light-matter interactions such as cavity quantum electrodynamics $(\mathrm{QED})^{1,2}$ and cavity polaritons, ${ }^{3,4}$ as well as various applications including vertical-cavity surface-emitting lasers (VCSELs), ${ }^{5-7}$ single-photon light sources, ${ }^{8}$ and Si-integrated on-chip lasers. ${ }^{9}$ Recently, it has been reported that the dispersion of a vertical cavity, i.e., the relationship between the frequency $\omega$ and wavevector $k$ of a cavity mode, can be engineered by using the high-index-contrast grating (HCG) as reflector and designing the dispersion of the HCG. ${ }^{10,11}$ Furthermore, a vertical-cavity in-plane (VCI) heterostructure can be formed in the $x$-direction by varying the HCG parameters, as shown in Fig. 1(a). ${ }^{12}$ In these reports, the discussions are focused on the dispersion profile in $k$-space along the $x$-direction. The $x$-direction represents a direction perpendicular to the grating lines, as defined in Fig. 1.

Here, we investigate the importance of anisotropic dispersion curvatures of HCG-based vertical cavities along the $x$ - and $y$-directions and illustrate their impacts on the properties of VCI heterostructures. For this study, the HCG parameters are varied in both $x$ - and $y$-directions, as shown in Fig. 1(b). The dispersion curvature is the second-order derivative of the frequency of a propagating mode with respect to the in-plane wavevector, and its inverse can be interpreted as an effective photon mass along the wavevector direction. As discussed below, the dispersion curvatures along both the $x$ - and $y$-directions can be engineered in the HCG-based cavities to have a specific positive, zero, or negative value. Furthermore, a heterostructure with significantly different dispersion curvatures (even different signs of curvatures) on each side of the heterostructure interface can be formed. Therefore, with HCG-based VCI heterostructures, one may design a heterostructure with an extra degree of freedom for the effective mass and control of the direction of energy flow. The control of the dispersion characteristics in the cavity may result in various well-known effects such as enhancing the spontaneous emission through the Purcell factor ${ }^{13}$ or controlling the properties of polariton lasers. ${ }^{14}$

${ }^{a)}$ Electronic mail: ilch@fotonik.dtu.dk
This paper is organised as follows. First, we derive an analytic expression for the dispersion curvature of a general cavity. This expression decomposes the cavity dispersion curvature into contributions from each mirror and the nominal cavity between the two mirrors. Using this expression, we discuss the properties of anisotropic dispersion curvatures. Then, we investigate three characteristic VCI photonic well structures to illustrate the rich physics of such structures. In the first and second structures, all the well and barrier regions have positive and negative cavity dispersion curvatures, respectively. These case studies explain how the order and spacing between the transverse mode frequencies are determined by the sign and magnitude of the cavity dispersion curvatures. As a potential application of these properties, the engineering of photon-photon resonances for achieving a high speed laser is discussed. In the third case, the well and barrier regions have opposite dispersion curvatures, which is not feasible in conventional heterostructures. Based on the insights obtained from these studies, we

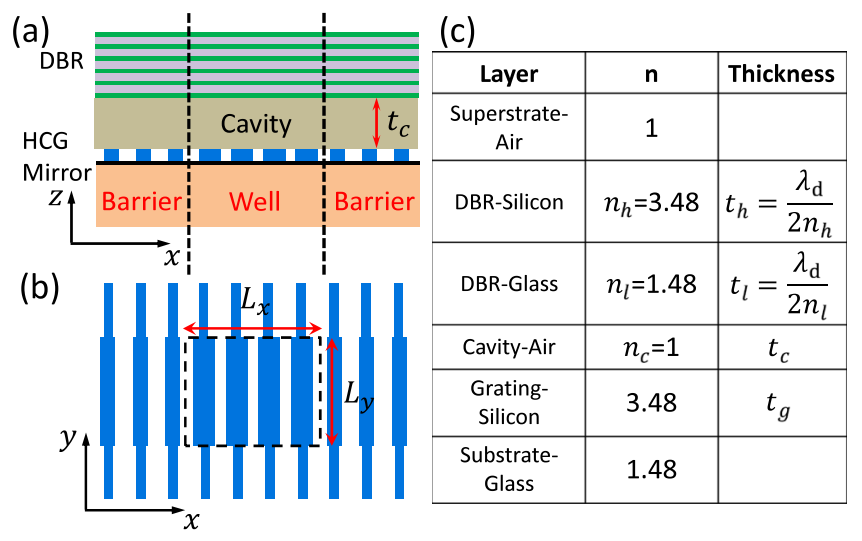

FIG. 1. (a) Cross-sectional schematic of the test structure consisting of a distributed Bragg reflector (DBR), a HCG mirror, and a low-refractive-index cavity layer. (b) Top view of the HCG layer. The grating bar width $W_{g}$ is varied along the $x$ - and $y$-directions to make a heterostructure with the well sizes of $L_{x}$ and $L_{y}$. (c) Refractive index and thickness of each layer. The grating parameters, $t_{g}$ (thickness), $\Lambda_{g}$ (period), and $W_{g}$ (width) are varied in different examples to obtain the required properties. Correspondingly, $t_{c}$ is changed to have a resonance at the design wavelength $\lambda_{d}$ of $1550 \mathrm{~nm}$. 
propose and analyze a system of laterally coupled vertical cavities. Compared to vertically coupled VCSELs, ${ }^{15}$ the coupling direction and light output/input direction are separated, which makes access to the properties of the individual cavities much easier. Using this system, the parity-time (PT) symmetry breaking phenomenon is reported in vertical cavities.

In Ref. 12, an expression for cavity dispersion curvature was discussed around the Fano resonance frequencies of the HCG comprising the vertical cavity. Here, we derive a general expression, which is valid at all frequencies within the HCG stopband and is in line with discussion in Ref. 10. In a vertical cavity, the mode frequency $\omega$ is found by solving the oscillation condition

$$
\begin{aligned}
& \phi_{1}\left(\omega, k_{x}, k_{y}\right)+\phi_{2}\left(\omega, k_{x}, k_{y}\right)-2 k_{z} t_{c}=2 m \pi, \\
& k_{z}=\sqrt{\left(\frac{n_{c} \omega}{c}\right)^{2}-k_{x}^{2}-k_{y}^{2}} .
\end{aligned}
$$

Here, $k_{x}, k_{y}$, and $k_{z}$ are wavevector components of a mode in the nominal cavity layer with a refractive index of $n_{c}$, and a thickness of $t_{c}$, and $c$ is the speed of light in vacuum. Note that the reflection phases from the two mirrors, $\phi_{1}$ and $\phi_{2}$, depend on the in-plane wavevectors, $k_{x}$ and $k_{y}$. The resonance frequency at a normal incidence, $\omega_{0}$, is determined by the following condition: $2 k_{z, 0} t_{c}=-2 m \pi+\phi_{1,0}+\phi_{2,0}$, where $k_{z, 0}=\omega_{0} n_{c} / c$ and $\phi_{i, 0}=\phi_{i}\left(\omega_{0}, 0,0\right)$.

The modes of our interest have a lateral extension of several times of wavelength. Thus, $k_{x}$ and $k_{y}$ distributions are close to the $\Gamma$ point where $\left(k_{x}, k_{y}\right)=(0,0) .{ }^{16}$ This validates the following Taylor expansion, keeping the first non-zero derivatives with respect to $k_{j}$ and $\omega$ :

$$
\phi_{i}\left(\omega, k_{x}, k_{y}\right) \simeq \phi_{i, 0}+\left.\frac{1}{2} \sum_{j=x, y} \frac{\partial^{2} \phi_{i}}{\partial k_{j}^{2}}\right|_{0} k_{j}^{2}+\left.\frac{\partial \phi_{i}}{\partial \omega}\right|_{0} \Delta \omega,
$$

where $\Delta \omega=\omega-\omega_{0}$ and the derivatives are evaluated at $\left(\omega, k_{x}, k_{y}\right)=\left(\omega_{0}, 0,0\right)$. Inserting Eq. (2) into Eq. (1) leads to

$$
\begin{aligned}
\omega & =\omega_{0}+\sum_{j=x, y} \beta_{j} k_{j}^{2}, \\
\beta_{j} & =\frac{c^{2}}{2 n_{c}^{2} \omega_{0}} \frac{t_{c}}{t_{\mathrm{eff}}}+\frac{c}{4 n_{c}} \frac{1}{t_{\mathrm{eff}}}\left(a_{1, j}+a_{2, j}\right),
\end{aligned}
$$

where $t_{\text {eff }}\left(=t_{c}+t_{1}+t_{c}\right)$ is the effective cavity thickness, $t_{i}$ $\left(=-\frac{c}{2 n_{c}} \partial \phi_{i} / \partial \omega\right)$ is the phase penetration into the $i$-th mirror, and $a_{i, j}=\partial^{2} \phi_{i} / \partial k_{j}^{2}$. The parameter $\beta_{j}$ represents the cavity dispersion curvature along the $j$-direction. The first term of $\beta_{j}$ results from the round-trip propagation in the nominal cavity and is always positive. The second term of $\beta_{j}$ accounts for the dispersion curvature from the mirrors. For HCGs, this mirror contribution can be either positive, negative, or even zero. Furthermore, it is polarization sensitive and anisotropic.

Using the derived expressions, we may elucidate the quantitative differences between different types of cavities. At a wavelength of $1550 \mathrm{~nm}$, the contribution of a $0.5 \lambda$-long nominal air cavity to the cavity dispersion curvature is approximately $30 \mathrm{~m}^{2} / \mathrm{s}$. As seen in Eq. (3), this nominal cavity contribution increases linearly with the cavity thickness, $t_{c}$. The mirror contribution of a typical distributed Bragg reflector (DBR) is on the order of $1 \mathrm{~m}^{2} / \mathrm{s}$ and gets smaller for larger refractive index contrast of the DBR layers. This mirror contribution is isotropic due to the rotational symmetry of DBR structures. Therefore, in DBRbased cavities, the cavity dispersion curvature is dominated by the nominal cavity contribution and is always positive and isotropic. Its shape resembles the conduction band of semiconductors. However, in the case of HCGs, the mirror contribution can be on the order of $\pm 100 \mathrm{~m}^{2} / \mathrm{s}$ or even larger. If the cavity thickness $t_{c}$ is small, e.g., less than $2 \lambda$, the cavity dispersion curvature of HCG-based cavities can be positive, zero, or negative, being dominated by the HCG dispersion curvature. Also, the cavity dispersion curvature is anisotropic along the $x$ - and $y$-directions and depends on the incident light polarization, as does the HCG mirror dispersion. Therefore, it is possible in HCG-based cavities to engineer the cavity dispersion by designing the phase response of the HCG while keeping its reflectivity high as discussed in Ref. 17. We note that the recently reported grating reflector, referred to as hybrid grating reflector ${ }^{18}$ or zero-contrast grating, ${ }^{19}$ has mirror dispersion properties similar to HCGs. The hybrid grating consists of a sub-wavelength grating layer and an unpatterned layer that may include a gain material.

The envelope approximation derived for photonic crystal $(\mathrm{PhC})$ heterostructures ${ }^{21,22}$ can be applied to analyze VCI heterostructures. ${ }^{23}$ The effective mass $m_{j}$ defined for the envelope approximation is related to the dispersion curvature, $\beta_{j}$ : $1 / m_{j}=\partial^{2} \omega^{2} / \partial k_{j}^{2}=4 \omega_{0} \beta_{j}$. Using the envelope approximation, the resonance frequency of a rectangular VCI photonic well $\omega_{p, q}$ is found to be $\mathrm{b}^{23}$

$$
\omega_{p, q}^{2} \simeq \omega_{0}^{2}+\frac{\alpha_{x}(p \pi)^{2}}{2 m_{x} L_{x}^{2}}+\frac{\alpha_{y}(q \pi)^{2}}{2 m_{y} L_{y}^{2}},
$$

where $p$ and $q$ are the mode numbers in the $x$ - and $y$-directions, respectively, $L_{x}$ and $L_{y}$ are the lengths of the heterostructure as defined in Fig. 1(b), and the rational factors $\alpha_{x}$ and $\alpha_{x}$ account for the effect of finite barrier heights. This expression will be used to interpret the result for the structures considered below.

To study the physics of VCI heterostructures, three characteristic photonic well structures are numerically investigated. The first structure has positive dispersion curvatures for both the well and barrier regions [Fig. 2(a)], the second one has negative dispersion curvatures for both the well and barrier [Fig. 2(b)], and the third has a mixture of positive and negative dispersion curvatures [Fig. 3(a)]. All structures have a $0.5 \lambda$-long air cavity, which can be formed by sacrificial etching in a similar way as in Ref. 23, and a HCG that is designed to be highly reflective for a TM polarized field. ${ }^{24}$ For simulations, an in-house developed three-dimensional (3D) simulator was used, ${ }^{25,26}$ which is based on a rigorous coupled wave analysis method ${ }^{27,28}$ and employs absorbing boundary conditions. ${ }^{29}$ The band edge profiles in Figs. 2-4 are found for each section of the VCI heterostructure, by assuming a grating that is periodic in the $x$-direction and infinitely long in the $y$-direction. 
(a)

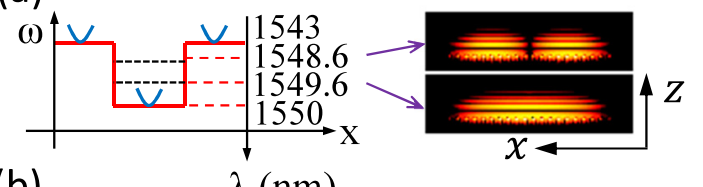

(b)

$\lambda(\mathrm{nm})$
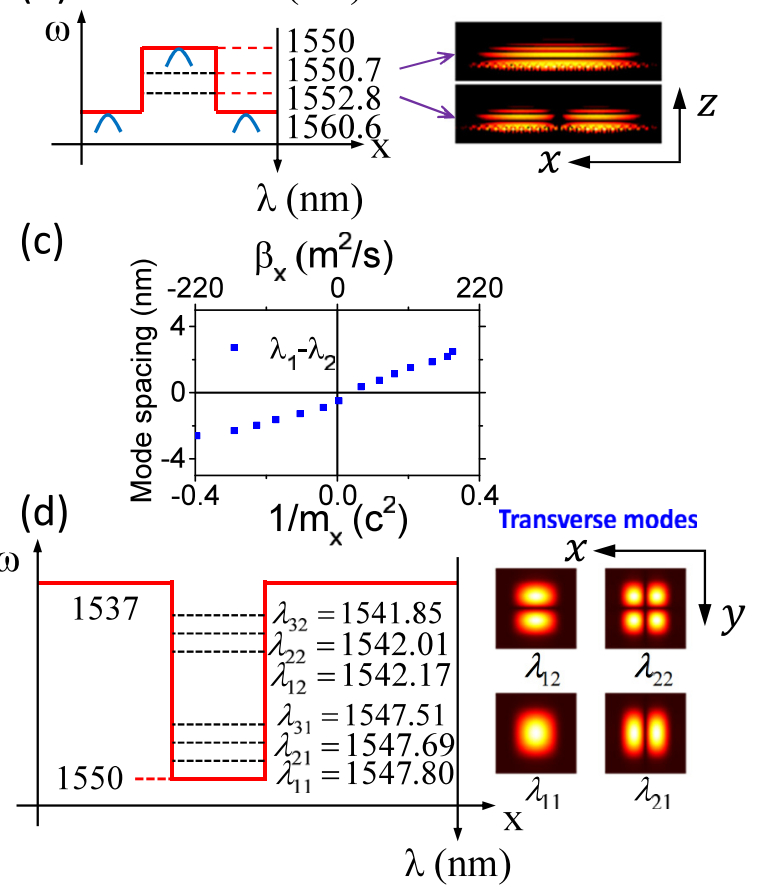

FIG. 2. Band edge profiles in red color for (a) the positive and (b) the negative dispersion curvatures, respectively. The first two transverse mode profiles are shown with their resonance wavelengths. Blue curves in (a) and (b) represent dispersion curves. (c) Transverse-mode wavelength spacing between the two lowest modes as a function of dispersion curvature. (d) Band edge profile of a 2D photonic well, and mode profiles therein obtained by $3 \mathrm{D}$ simulations. This explains the mode grouping effect in a $2 \mathrm{D}$ photonic well due to different effective masses in the $x$ - and $y$-directions. All simulation parameters can be found in Ref. 20.

It is well known in the VCSEL literature that higher order transverse modes have higher frequencies, i.e., shorter wavelengths, due to their higher in-plane wavevectors. However, the in-plane dispersion of HCG structures can significantly modify these characteristics. For the

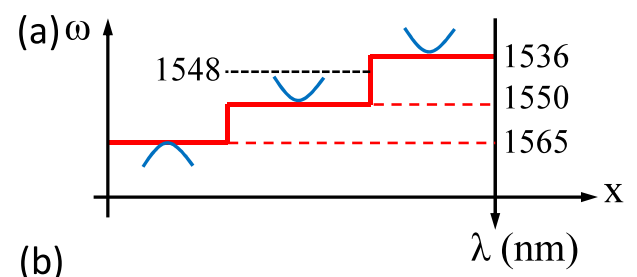

(b)

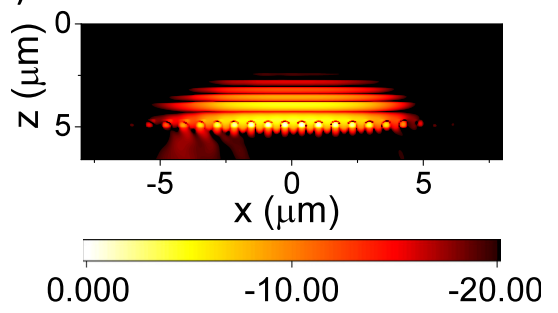

FIG. 3. (a) Band edge profile of a photonic well structure with opposite effective masses in the left and right barriers. (b) Normalized field profile $\left(\left|H_{y}\right|\right)$ of the structure in (a) (dB scale). All simulation parameters can be found in Ref. 20. photonic wells with positive dispersion curvatures, we have the usual situation of VCSELs, as shown in Fig. 2(a): the fundamental mode has the longest wavelength. However, for the negative dispersion curvature case, the fundamental mode has a shorter wavelength than the higher order mode, as shown in Fig. 2(b). Referring to Eq. (4), this observation can be interpreted like this: the higher order mode with more spatial modulation adds a larger negative kinetic energy, lowering the total energy. The positive dispersion curvature (electron-like) and the negative dispersion curvature (hole-like) cases are analogous to the electronic quantum wells in the conduction band and valence band, respectively. This observation is generalized in the result shown in Fig. 2(c).

Figure 2(c) plots the wavelength spacing of the two lowest transverse modes as a function of the $x$-direction dispersion curvature $\beta_{x}$, in the well region. The grating parameters are kept constant, and the design wavelength is varied across the HCG stop-band to change the effective mass. ${ }^{20}$ Therefore, the mode size is nearly identical in the investigated parameter range. It shows that the wavelength spacing increases with the dispersion curvature. This observation can be understood also by referring to Eq. (4): with a smaller dispersion curvature, corresponding to a larger effective mass, the kinetic energy contribution to the total energy becomes smaller, leading to a smaller energy difference between the two transverse modes. Therefore, the transverse mode spacing can be controlled by engineering the dispersion of the HCG, without changing the size of the transverse mode. This results in interesting phenomena such as mode grouping and mode degeneracy, with several possible applications, as discussed below.

If $m_{x}$ and $m_{y}$ largely differ, the transverse modes are grouped as shown in Fig. 2(d). In Fig. 2(d), the effective mass along the $y$-direction is $\sim 10$ times smaller than that along the $x$-direction. As a result, the second mode number for the $y$ direction determines the larger wavelength spacing between groups, while the first one for the $x$-direction determines the smaller wavelength spacing within a group. This mode grouping is experimentally observed in a HCG-DBR cavity laser, ${ }^{23}$ and the 3D simulations give the same result. The wavelengths and mode profiles in Fig. 2(d) are obtained by the 3D simulations. Furthermore, the fundamental mode frequency $\omega_{0,0}$ and the higher order mode frequencies $\omega_{p, p}$ can be made degenerate, by designing the effective mass, so that $m_{x}=-m_{y}$ and $\alpha_{x} / L_{x}^{2}=\alpha_{y} / L_{y}^{2}$ [cf. Eq. (4)]. This transverse mode-degeneracy is also confirmed by the $3 \mathrm{D}$ simulations.

This control over the transverse mode spacings can be used to boost the speed of a diode laser. Recently, the bandwidth boost of the laser diodes has attracted a lot of attention. ${ }^{30-32}$ The boost mechanism is based on the introduction of a photon-photon resonance at a frequency higher than the relaxation oscillation frequency by exploiting external optical feedback or cross-gain modulation. In HCG-based vertical cavities, multiple transverse modes can be designed to have specific wavelength spacings, e.g., $0.15 \mathrm{~nm}$, which determine the photon-photon resonance frequency. In this way, multiple photon-photon resonances can be introduced at designed frequencies through cross-gain modulation. For this, all the involved transverse modes need to be lasing, which is 
feasible in the HCG-based cavities since we can separately control the mode profile and the gain profile. Furthermore, external optical feedback in the in-plane directions may be introduced to tailor the frequency response function. Recently, we have demonstrated a high-speed Si-integrated vertical cavity laser, using the in-plane optical feedback. ${ }^{33}$

The unique possibility to design various effective masses in the well and barrier regions enables exotic photonic well configurations. An interesting example is a photonic well where the sign of effective mass in the barrier region is opposite to that of the well region, e.g., hole-like barrier with electron-like well. In order to obtain the transverse mode confinement, the band edge of the barrier should be lower than that of the well. This band edge alignment is opposite to the case shown in Fig. 2(a), where both the barrier and well are electron-like cavities. To compare these two cases, we investigate a VCI heterostructure with positive effective masses in the well and right barrier and a negative effective mass for the left barrier, as illustrated in Fig. 3(a). The mode profile is well confined within the well section, as shown in Fig. 3(b). If the band edge of the left barrier is moved above the band edge of the well, the field is no longer confined in the well. This example shows that HCG-based VCI heterostructures allow more freedom for designing the photonic wells by controlling the effective mass as well as the band edge.

Coupled micro-cavity structures have displayed many interesting phenomena, such as miniband formation, ${ }^{34}$ heavy photons, ${ }^{35}$ coupled-cavity QED, ${ }^{36}$ and recently PT symmetry breaking. ${ }^{37,38}$ For the implementation of these, various structures have been suggested, including $\mathrm{PhC}$ coupled cavities, ${ }^{34}$ microring resonators, ${ }^{37,38}$ and vertically coupled VCSELs. ${ }^{15}$ Here, we propose laterally coupled vertical cavities, as shown in Fig. 4(a). We note that, in the proposed coupled cavities, the directions of the light propagation (vertical) and coupling (lateral) are separated from each other, while they are in the same directions in other coupled cavities. This

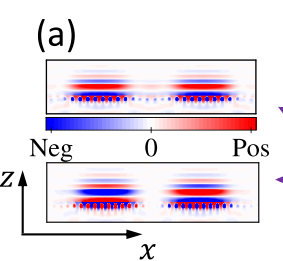

(b)

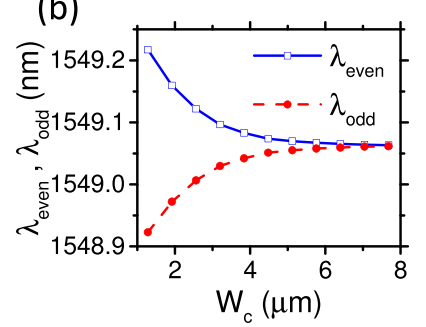

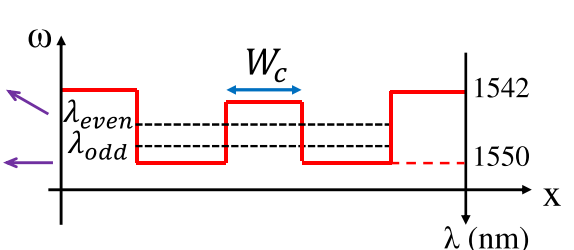

(c)

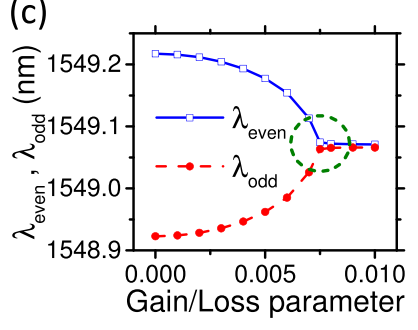

FIG. 4. (a) Band-edge profile of laterally coupled vertical cavities and the mode profiles (real value of $H_{y}$ ) of even and odd modes. (b) Wavelengths of the even and odd modes versus lateral spacing between the two cavities. (c) Wavelengths of the even and odd modes as function of gain/loss parameters, which are the imaginary part of refractive indices of quantum wells. The exceptional point, as indicated by a green dotted circle, shows the braking of PT-symmetry in the structure by increasing the gain/loss parameter. All simulation parameters can be found in Ref. 20 . makes access to the properties of the individual cavities easier. As shown in Fig. 4(a), the coupling of two identical cavities leads to two coupled states with even and odd parities. The coupling strength can be tuned by changing the barrier width, height, or effective mass. Here, we choose the barrier width $W_{c}$. As shown in Fig. 4(b), the separation between the wavelengths of the two resulting states becomes larger for a larger coupling, i.e., smaller $W_{c}$. As shown in Fig. 4(c), the PT symmetry of these coupled cavities can be broken by introducing a gain region in one cavity and a loss region in the other cavity, which reduces the wavelength separation. The exceptional point, as indicated by a green dotted circle, is a characteristic signature of PT-symmetry breaking. ${ }^{37-40}$ The exceptional point does not show very steep (perpendicular) bifurcation observed in the ideal PT-symmetry broken case. ${ }^{37,39,41}$ This is due to the unbalanced total gain/loss in the system as explained in Refs. 39 and 42.

In conclusion, the in-plane dispersion curvatures of the HCG-based vertical cavities can be designed to achieve different values in different directions. We have shown that this enables the engineering of heterostructures with exotic properties that may be of interest for various applications. As an example, a system of two laterally coupled vertical cavities has been proposed and investigated, which exhibits the spontaneous breaking of parity-time symmetry.

The authors gratefully acknowledge the support from the Danish Council for Independent Research (Grant No. 0602-01885B), the Innovation Fund Denmark through the HOT project (Grant No. 5106-00013B), as well as Villum Fonden via the NATEC Centre of Excellence.

${ }^{1}$ J. Gérard, B. Sermage, B. Gayral, B. Legrand, E. Costard, and V. ThierryMieg, Phys. Rev. Lett. 81, 1110 (1998).

${ }^{2}$ K. J. Vahala, Nature 424, 839 (2003).

${ }^{3}$ H. Deng, G. Weihs, C. Santori, J. Bloch, and Y. Yamamoto, Science 298, 199 (2002).

${ }^{4}$ S. Christopoulos, G. B. H. Von Högersthal, A. Grundy, P. Lagoudakis, A. Kavokin, J. Baumberg, G. Christmann, R. Butté, E. Feltin, J.-F. Carlin et al., Phys. Rev. Lett. 98, 126405 (2007).

${ }^{5}$ P. Moser, J. Lott, P. Wolf, G. Larisch, A. Payusov, N. N. Ledentsov, and D. Bimberg, IEEE J. Sel. Top. Quantum Electron. 19, 7900406 (2013).

${ }^{6}$ P. Westbergh, J. S. Gustavsson, A. Haglund, M. Sköld, A. Joel, and A. Larsson, IEEE J. Sel. Top. Quantum Electron. 15, 694 (2009).

${ }^{7}$ H. Dalir and F. Koyama, Appl. Phys. Lett. 103, 091109 (2013).

${ }^{8}$ M. Pelton, C. Santori, J. Vucković, B. Zhang, G. S. Solomon, J. Plant, and Y. Yamamoto, Phys. Rev. Lett. 89, 233602 (2002).

${ }^{9}$ I.-S. Chung and J. Mørk, Appl. Phys. Lett. 97, 151113 (2010).

${ }^{10}$ Z. Wang, B. Zhang, and H. Deng, Phys. Rev. Lett. 114, 073601 (2015).

${ }^{11}$ A. Taghizadeh, J. Mork, and I.-S. Chung, CLEO: Science and Innovations (Optical Society of America, 2015), p. SW1F-4.

${ }^{12}$ C. Sciancalepore, B. B. Bakir, X. Letartre, J.-M. Fedeli, N. Olivier, D. Bordel, C. Seassal, P. Rojo-Romeo, P. Regreny, and P. Viktorovitch, J. Lightwave Technol. 29, 2015 (2011).

${ }^{13}$ E. M. Purcell, Phys. Rev. 69, 37 (1946).

${ }^{14}$ B. Zhang, S. Brodbeck, Z. Wang, M. Kamp, C. Schneider, S. Höfling, and H. Dening, Appl. Phys. Lett. 106, 051104 (2015).

${ }^{15}$ R. Stanley, R. Houdre, U. Oesterle, M. Ilegems, and C. Weisbuch, Appl. Phys. Lett. 65, 2093 (1994).

${ }^{16}$ A. Liu, W. Hofmann, and D. Bimberg, Opt. Express 22, 11804 (2014).

${ }^{17}$ L. Carletti, R. Malureanu, J. Mørk, and I.-S. Chung, Opt. Express 19, 23567 (2011).

${ }^{18}$ A. Taghizadeh, G. C. Park, J. Mørk, and I.-S. Chung, Opt. Express 22, 21175 (2014).

${ }^{19}$ R. Magnusson, Opt. Lett. 39, 4337 (2014). 
${ }^{20}$ See supplementary material at http://dx.doi.org/10.1063/1.4935084 for detailed simulation parameters.

${ }^{21}$ M. Charbonneau-Lefort, E. Istrate, M. Allard, J. Poon, and E. H. Sargent, Phys. Rev. B 65, 125318 (2002).

${ }^{22}$ E. Istrate and E. H. Sargent, Rev. Mod. Phys. 78, 455 (2006).

${ }^{23}$ G. C. Park, W. Xue, A. Taghizadeh, E. Semenova, K. Yvind, J. Mørk, and I.-S. Chung, Laser Photonics Rev. 9, L11 (2015).

${ }^{24}$ I.-S. Chung, Opt. Express 23, 16730 (2015).

${ }^{25}$ A. Taghizadeh, J. Moerk, and I.-S. Chung, Metamaterials (IEEE, 2014), pp. 277-279.

${ }^{26}$ A. Taghizadeh, J. Mørk, and I.-S. Chung, Opt. Express 23, 14913 (2015).

${ }^{27}$ M. Moharam, T. Gaylord, E. B. Grann, and D. A. Pommet, J. Opt. Soc. Am. 12, 1068 (1995).

${ }^{28}$ L. Li, J. Opt. Soc. Am. 14, 2758 (1997).

${ }^{29}$ J. P. Hugonin and P. Lalanne, J. Opt. Soc. Am. 22, 1844 (2005).

${ }^{30}$ O. Graydon, Nat. Photonics 9, 75 (2015).

${ }^{31}$ S. Mieda, S. Shiratori, N. Yokota, W. Kobayashi, and H. Yasaka, Appl. Phys. Express 8, 022701 (2015).

${ }^{32}$ H. Dalir and F. Koyama, Appl. Phys. Express 7, 022102 (2014).
${ }^{33}$ G. C. Park, W. Xue, M. Piels, D. Zibar, J. Mørk, E. Semenova, and I.-S. Chung, "Ultrahigh-speed Si-integrated on-chip laser with tailored dynamic characteristics," Sci. Rep. (submitted).

${ }^{34}$ T. D. Happ, M. Kamp, A. Forchel, J.-L. Gentner, and L. Goldstein, Appl. Phys. Lett. 82, 4 (2003).

${ }^{35}$ M. Bayindir and E. Ozbay, Phys. Rev. B 62, R2247 (2000).

${ }^{36}$ S. Hughes, Phys. Rev. Lett. 98, 083603 (2007).

${ }^{37}$ B. Peng, Ş. K. Özdemir, F. Lei, F. Monifi, M. Gianfreda, G. L. Long, S. Fan, F. Nori, C. M. Bender, and L. Yang, Nat. Phys. 10, 394 (2014).

${ }^{38}$ B. Peng, S. Özdemir, S. Rotter, H. Yilmaz, M. Liertzer, F. Monifi, C. Bender, F. Nori, and L. Yang, Science 346, 328 (2014).

${ }^{39}$ C. M. Bender, M. Gianfreda, S. K. Özdemir, B. Peng, and L. Yang, Phys. Rev. A 88, 062111 (2013).

${ }^{40}$ H. Ramezani, H.-K. Li, Y. Wang, and X. Zhang, Phys. Rev. Lett. 113, 263905 (2014).

${ }^{41}$ M. Brandstetter, M. Liertzer, C. Deutsch, P. Klang, J. Schöberl, H. Türeci, G. Strasser, K. Unterrainer, and S. Rotter, Nat. Commun. 5, 4034 (2014).

${ }^{42}$ H. Benisty, C. Yan, A. Degiron, and A. Lupu, J. Lightwave Technol. 30, 2675 (2012). 